\title{
Spectrum reconstruction for Chang'e-1 imaging interferometer data using modified periodogram method
}

\author{
Feng Zhu ${ }^{1,2,3, \dagger}$ Jia-Hang Liu ${ }^{1}$, Xin Ren ${ }^{2}$, Tie-Qiao Chen ${ }^{1}$ and Jia Liu ${ }^{1}$, \\ ${ }^{1}$ Laboratory of Remote Sensing and Intelligent Information System, Xi'an \\ Institute of Optics and Precision Mechanics, Chinese Academy of Sciences, \\ $X i$ 'an, Shaanxi 710119, China \\ ${ }^{2}$ Key Laboratory of Lunar and Deep Space Exploration, \\ National Astronomical Observatories, Chinese Academy of Sciences, \\ Beijing 100012, China \\ ${ }^{3}$ National Space Science Center, University of Chinese Academy of Sciences, \\ Beijing 100190, China \\ ${ }^{\dagger}$ E-mail: zhufeng@opt.ac.cn
}

\begin{abstract}
Imaging Interferometer (IIM) aboard on Chang'e-1 is a Fourier transform imaging spectroscopy. Spectrum reconstruction which converts raw interferometric data to spectral data is crucial to the signal-to-noise (SNR) property and spectral consistency, thus influences the usability of IIM data. Three modified periodogram method including periodogram with rectangular window (PRW), periodogram with triangular window (PTW) and Bartlett method are introduced for the spectrum reconstruction. We use the Spectral and Spatial Decorrelation method to calculate the SNRs, and use Apollo samples to calibrate the spectrum and validate the spectral consistency of the three methods. Experimental results showed that Bartlett method had better performance in both SNR and spectral consistency than other two methods.
\end{abstract}

Keywords: Chang'e-1; Imaging Interferometer; Spectrum Reconstruction; Periodogram; Bartlett

\section{Introduction}

China's first lunar Chang'e-1 was launched on October 24, 2007. Imaging Interferometer (IIM), as one of the eight scientific payloads embarked on Chang'e-1, is a Fourier transform Sagnac-based imaging spectrometer with goals to analyze and map the abundance and distribution of various chemical elements on the lunar surface[1]. IIM data has already shown its potentials in lunar elemental mappings and geologic studies [2-6]. However, IIM's spectral data suffers from several degradation problems. The first and last few bands has poor SNRs which reduces the usability of IIM data. In some previous study, the degraded bands are often discarded [2,3].

Spectrum reconstruction converting raw interferometric data to spectral data is crucial to the quality of spectral data. In essence, spectrum reconstruction is a 
solution to estimate how radiance is distributed over wavelength from a finite record of interferometric data sequence. Periodogram is a common-used tool to estimate spectra. While the periodogram method has high statistical variability, the modified methods that have lower variance at a cost of reduced resolution were developed, such as Blackman-Tukey method, Bartlett method, and Welch method [8].

The paper is organized as follows: Section 2 gives a brief description about the principle of Imaging Interferometer and spectrum reconstruction. Then Section 3 introduces three modified periodogram methods. In Section 4, several experiments are performed to compare the SNRs and spectral consistency of the three different methods. Finally, the conclusions are presented in Section 5.

\section{Principle of Imaging Interferometer}

IIM aboard on Chang'e-1 is a Sagnac-based spatially modulated imaging interferometer [9], the optical principle schematic of which is shown in Fig. 1. The IIM mainly consists of fore-optics, Sagnac interferometer, Fourier lens, cylindrical lens, CCD detector and signal processing system. The fore-optics converges the reflected radiation from the target and forms the image at its focal plane. Through the slit, the radiation is then sheared into two beams by the Sagnac interferometer (also known as common path interferometer). Two beams follow the same path but in opposite direction (one in reflectance and the other in transmission), as though they come from two virtual coherent sources. Since the sources are at the front local plane of the Fourier lens, the beam emerging from the lens is collimated at the back focal plane. The cylindrical lens placed between Fourier lens and CCD detector allows the spatially resolved observation of target along the slit. Eventually, the CCD detector acquires the interferogram (shown in Fig. 2b), each row of which corresponds to the interference fringe (shown in Fig. 2c) emitted from different position of slit. The interferogram contains space information cross the track and spectral information. With the movement of IIM, another space information along the track will be acquired. By the data processing known as spectrum reconstruction, the spectrum information in the spatial domain can be restored (shown in Fig. 2a).

According to the principle of the IIM, the spectrum reconstruction which converts the interferometric information into spectrum information is a solution to estimate how radiance is distributed over wavelength from a finite record of interferometric data sequence. 


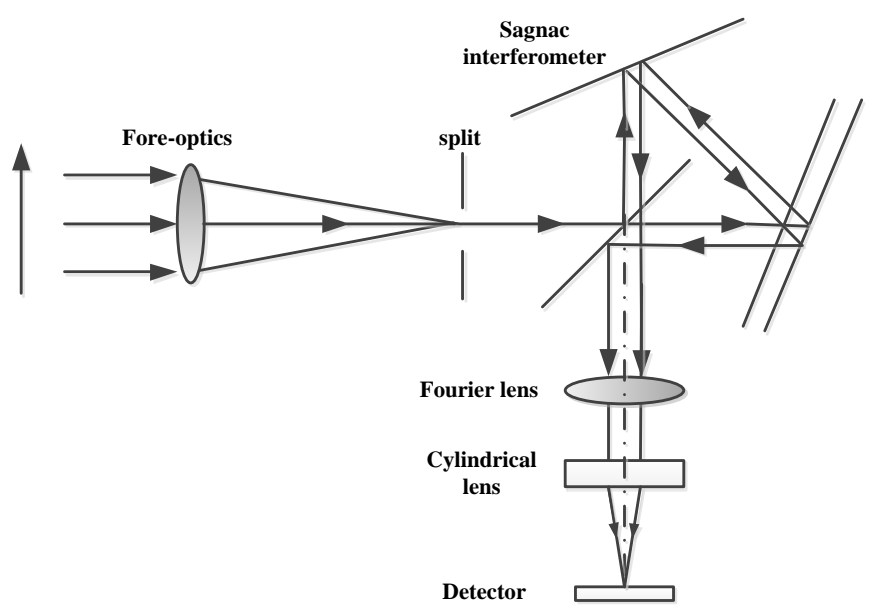

Fig. 1. The optical principle schematic of Imaging Interferometer (IIM).

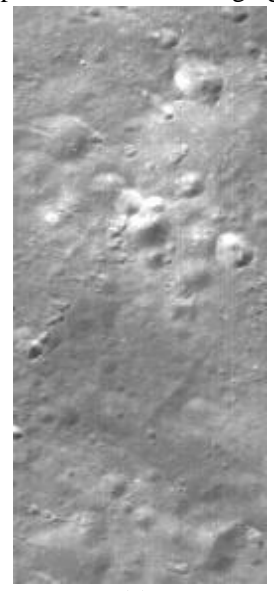

(a)

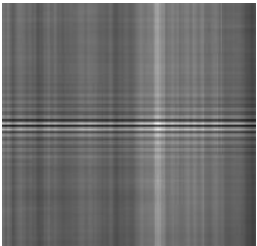

(b)

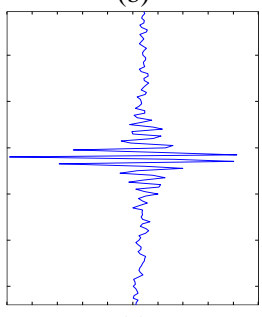

Fig. 2. Illustration of spectrum reconstruction. (a) reconstructed lunar image; (b) interferogram; (c) the interference stripe of the first sample of the interferogram image.

\section{The Modified Periodogram Method}

Periodogram is one of the most common-used methods for spectral estimation, the definition of which is given by:

$$
\hat{\phi}_{p}(\omega)=\frac{1}{N}\left|\sum_{t=1}^{N} y(t) e^{-i \omega t}\right|^{2}
$$

where $\{y(t) ; t=1,2, \ldots, N\}$ denote a discrete data sequence, specifically the interferometric data sequence in this paper (shown in Fig. 2c). 
The main problem with the periodogram is the high statistical variability of estimated spectrum. This effects may be reduced by apodization of the data sequence with window function $w(t)$ and then periodogram is modified as follows:

$$
\hat{\phi}_{p w}(\omega)=\frac{1}{N}\left|\sum_{t=1}^{N} y(t) w(t) e^{-i \omega t}\right|^{2}
$$

To further reduce the statistical variability of periodogram, we split up the $N$ samples into $L=N / M$ subsamples of $M$ samples each, and then average the periodograms obtained from the subsamples. This is called Bartlett method. Mathematically, the Bartlett method can be described as follows. Let

$$
y_{j}(t)=y((j-1) M+t)
$$

denote the observations of the $j$-th subsamples, and let

$$
\hat{\phi}_{j}(\omega)=\frac{1}{M}\left|\sum_{t=1}^{M} y_{j}(t) e^{-i \omega t}\right|^{2}
$$

denote the corresponding periodogram. The estimated spectrum using Bartlett method is then given by:

$$
\hat{\phi}_{B}(\omega)=\frac{1}{L} \sum_{j=1}^{L} \hat{\phi}_{j}(\omega)
$$

Note that the modified periodogram methods decrease the variance of the estimated spectrum at the expense of decreasing the average resolution. Under the guarantee of reasonable spectral resolution, the modified methods can be reliable and effective in spectrum reconstruction for IIM data.

\section{Results and Discussion}

Three common kinds of periodogram method including periodogram with rectangular window (PRW), periodogram with triangular window (PTW) and Bartlett method were used to perform the spectrum reconstruction for IIM data. For comparison we perform two sets of contrast experiments to evaluate the signal-to-noise (SNR) property and spectral consistency of the three methods.

\subsection{Signal-to-Noise experiments}

The estimation of SNR is often influenced by geological condition. To assure the reliability of estimated SNR, the sub-image of $420 \times 128$ pixels corresponding to line $8000-8419$ and sample $1-128$ of IIM orbit 2212 is selected, which is 
relatively homogeneous in geology. After spectrum reconstruction using the three methods, we obtain spectrum data cube of 32 spectral bands ranging from $480 \mathrm{~nm}$ to $960 \mathrm{~nm}$. The Spectral and Spatial Decorrelation (SSDC) method is chosen for estimating signal-to-noise of the three sets of spectrum data quantitatively, considering that SSDC has been extensively used for estimating noise in hyperspectral images such as AVIRIS $[9,10]$.

The SNRs of three sets of spectrum data are shown in Fig. 3. The SNRs of spectrum date using PRW method are minimum at most of the 32 bands, especially at short wavelength and long wavelength. PTW method has better performance especially in middle bands while the SNRs are still not satisfactory (less than 20) at the both ends of wavelength. The SNRs of spectrum data using Bartlett method are basically equivalent at whole bands and maximum at half bands (16 bands). Above all, the SNRs at short and long wavelength get considerable improvements by using Bartlett method.

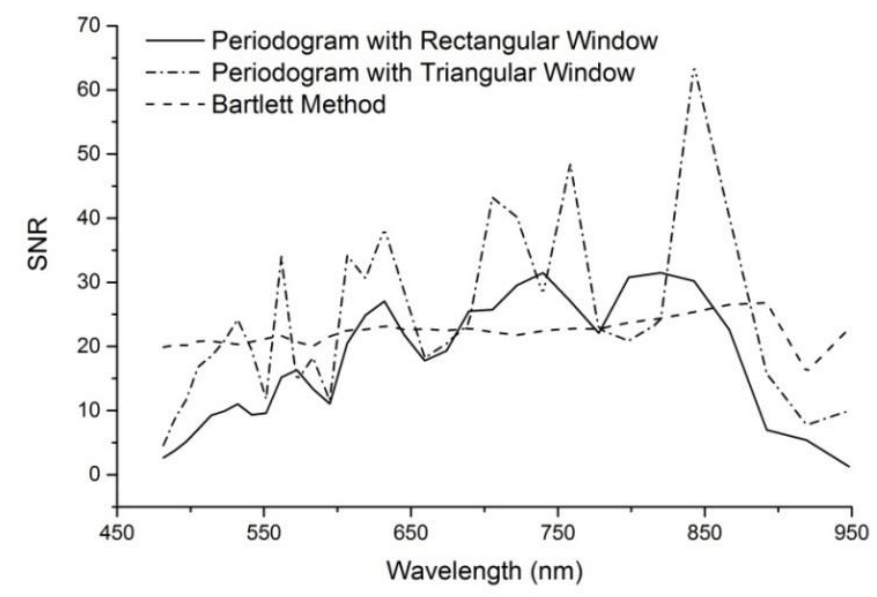

Fig. 3. The SNR of IIM spectral image obtained from three different methods.

In previous study, such as material identification and land cover classification, the first and last few bands of IIM data are often discarded for their low SNRs. The degraded image quality will lead to misleading interpretations and inaccuracy of applications. However, the information contained in the first and last few bands is quite valuable. For example, the 31th band $(918.1 \mathrm{~nm})$ is used to replace the last band $(946.6 \mathrm{~nm})$ to map the global distribution of chemical composition from IIM data [2], although the last band is the nearest band to the $950 \mathrm{~nm}$ band applied in Clementine data. Furthermore, we illustrate the comparison of image quality of the last band using the three methods respectively. As shown in Fig. 4, Bartlett method has excellent performance in the image quality because of its lowest statistical variability. 
Then follows the PTW method. The quality of image obtained from PRW method behaves so poor that we can barely identify any objects from the image.

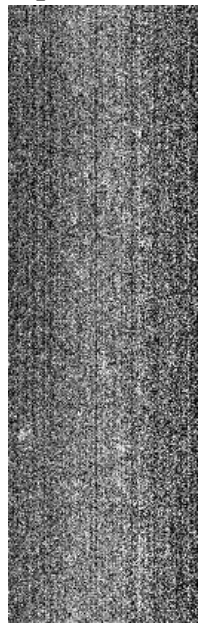

(a)

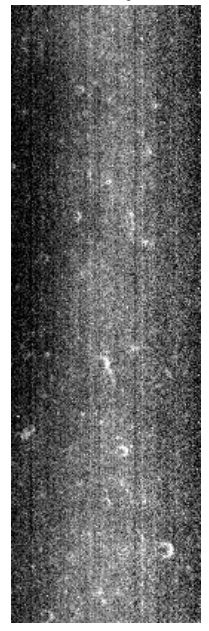

(b)

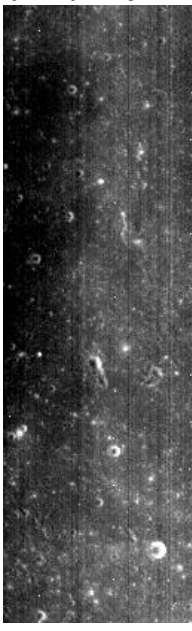

(c)

Fig. 4. The 32th band of IIM spectral image reconstructed using different methods: (a) periodogram with rectangular window; (b) periodogram with triangular window; (c) Bartlett method

\subsection{Spectral consistency experiments}

After spectrum reconstruction, we obtain the relative spectral intensity of target. Then absolute radiometric calibration is applied to get the absolute radiance. The coefficients for absolute radiometric calibration is prepared by pre-flight performance tests. Actually, only the coefficients of PRW method are acquired while the coefficients of the other two methods are unknown. So for credibility of comparing the spectral consistency of the three methods, the spectral intensity is calibrated with modeled spectra. The reflectance spectra of Apollo 16 sample 62231 has been extensively used as an optical standard for the calibration of lunar remote sensing data including the Clementine UVVIS, Kaguya MI and Chandrayaan-1 $\mathrm{M}^{3}$ [5]. The radiometric calibration is conducted as follows: (a) An calibration area which is near sample 62231 was chosen in IIM Orbit 2225 containing $5 \times 18$ pixels (sample: 124-128; line: 11117-11134). The average spectra of this area was computed and denoted as $D N_{\text {samp }}$. (b) Resample the spectra of sample 62231 to the IIM's bands using spline interpolation, denoted as $R_{\text {samp. }}$ (c) The calibration coefficients for each was computed to convert the IIM's spectral intensity to reflectance by eq.(6), where the $R_{\mathrm{IIM}}$ represents the reflectance of IIM and $\boldsymbol{D} \boldsymbol{N}_{\text {IIM }}$ represents the intensity of IIM.

$$
R_{\text {IIM }}=D N_{\text {IIM }} \times R_{\text {samp }} / D N_{\text {samp }}
$$


In this paper, we choose Apollo 17 Sample 71501 to validate the spectral consistency of IIM. The corresponding area locate in IIM's Orbit 2212 containing $5 \times 5$ pixels (sample: 90-94; line: 6825-6829). The resampled reflectance of Apollo 17 Sample 71501 and the calculated reflectance by eq.(6) of three periodogram methods are shown in Fig. 5. The waveform of the reflectance obtained from Bartlett method keeps consistent with that of resampled Apollo 17 Sample 71501 basically and has low fluctuation at short bands. Meanwhile, the deviation of reflectance at last band obtained from Bartlett method is minimum.

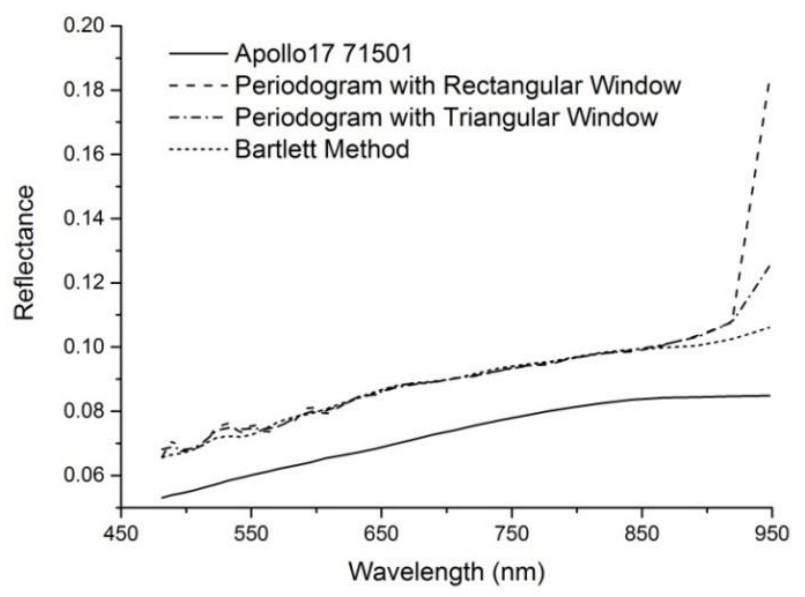

Fig. 5. The reflectance curve of Apollo 17 sample 71501 and IIM data from three methods

Note that all the three reflectance curves obtained from modified periodogram methods are somewhat above the resampled reflectance curve of Apollo sample. This is probably because there exist differences between the remotely measured reflectance values and those of returned Apollo samples measured in the laboratory, as texture and microstructure of these samples had been inevitably altered during sampling process[11]. In addition, the correction of geographic location may be not accurate enough.

\section{Conclusion}

In this paper, three different modified periodogram methods were introduced for spectrum reconstruction of IIM data. Bartlett method has the lowest estimated variability by theoretical analysis. The subsequent experiments also prove that Bartlett has a better performance in both SNR property and spectral consistency, especially at the short and long bands. This will evidently improve the usability of the short and long bands of IIM data. Further work such as mineral mapping using IIM data reconstructed from modified periodogram method hopes to validate the improvement of the usability of IIM data. 


\section{Acknowledgments}

The Chang'e-1 IIM data were provided by the Ground Segment for Data, Science and Application (GSDSA) of China's Lunar Exploration Program. This work was jointly supported by the Open Fund of Key Laboratory of Lunar and Deep Space Exploration of CAS, the Western Youth Scholars Program [Grant No. XAB2015A07] and the Open Fund of Key Laboratory of Spectral Imaging Technology of CAS [Grant No. LSIT201406].

\section{References}

1. Z. Ouyang, J. Jiang, L. I. Chunlai, et al, Preliminary Scientific Results of Chang'E-1 Lunar Orbiter: Based on Payloads Detection Data in the First Phase, Chin J Space Sci 28, 361-369 (2008).

2. B. Yan, S. Q. Xiong, Y. Wu, et al, Mapping Lunar global chemical composition from Chang'E-1 IIM data, Planetary \& Space Science 67, 119129 (2012).

3. Y. Wu, S. Besse, J. Y. Li, et al, Photometric correction and in-flight calibration of Chang'E-1 Interference Imaging Spectrometer (IIM) data, Icarus 222, 283-295 (2013).

4. Ling Z. C, Zhang J, Liu J.Z, et al, Lunar global FeO and $\mathrm{TiO}_{2}$ mapping based on the recalibrated Chang'E-1 IIM dataset, Acta Petrologics Sinica 32 87-98 (2016).

5. F. J. Liu, L. Qiao, Z. Liu, et al, Estimation of lunar titanium content: Based on absorption features of Chang'E-1 interference imaging spectrometer (IIM), Science China Physics Mechanics \& Astronomy 53, 2136-2144 (2010).

6. Z. Jiang, Z. C. Ling, J. Z. Liu, et al, Lunar absolute reflectance as observed by Chang'E-1 Imaging Interferometer, Science China Physics Mechanics \& Astronomy 58, 1-6 (2015).

7. B. C. Zhao, J. F. Yang, B. Xue, et al, Calibration of Chang'E-1 Satellite Interference Imaging Spectrometer, Acta Photonica Sinica 39, 769-775 (2010).

8. R. L. M. P. Stoica, "Introduction of Spectral Analysis," (1997).

9. R. E. ROGER, and J. F. ARNOLD, "Reliably estimating the noise in AVIRIS hyperspectral images," International Journal of Remote Sensing 17, 1951-1962 (1996).

10. Liu Bin, Ling Zongcheng, Liu Jianzhong, et al. "Image quality assessment for Chang'E-1 IIM 2C data.” Chin. J.Space Sci. 32, 440-448 (2012).

11. Pieters C. M, Boardman J. W, Ohtake M, et al, "One Moon, many measurements 1: Radiance values”, Icarus 226, 951-963 (2013). 